\title{
Cell seeding chamber for bone graft substitutes
}

\author{
J. Hennig ${ }^{1}$, M. Schieker ${ }^{2}$, H. Seitz ${ }^{1}$, \\ ${ }^{1}$ Fluid Technology and Microfluidics, University of Rostock, Rostock, Germany, joern.hennig@uni-rostock.de \\ ${ }^{2}$ Laboratory for Experimental Surgery and Regenerative Medicine, Department of Surgery, Ludwig Maximilians Uni- \\ versity, Munich, Germany
}

\begin{abstract}
There is an increasing demand for bone graft substitutes that are used as osteoconductive scaffolds in the treatment of bone defects and fractures. Achieving optimal bone regeneration requires initial cell seeding of the scaffolds prior to implantation.

The cell seeding chamber is a closed assembly. It works like a sandglass. The position of the scaffold is between two reservoirs containing the fluid (e.g. blood). The fluid at the upper reservoir flows through the scaffold driven by gravity. Fluid is collected at the lower reservoir. If the upper reservoir is empty the whole assembly turned and the process starts again.
\end{abstract}

A new compact cell seeding chamber for initial cell seeding has been developed that can be used in the operating theater.

\section{Introduction}

Because of the demographic change clinicians will be more and more confronted with diseases of mature people. Critical size bone defects are one of these problems. In more than $40 \%$ of these cases autologous bone graft substitutes are not available [1]. In spite of the high healing potential of the human body a permanent loss of bone tissue is still a unsolved complex clinical problem [1][2]. The implantation of autologous bone is currently the "golden standard" [10]. The disadvantage is the surgery which is necessary to extract the implant and the limited availability. Allogenic, xenogenic and synthetic bone graft substitutes are basically "death" structures. The healing behavior of these non living structures is insufficient. To improve the healing behavior it is necessary to vitalize the scaffolds with body's own cells [8]. These cells are contained in blood or bone marrow for example.

Basically there are two systems or methods to vitalize nonliving scaffolds. On the one hand there are systems for laboratory use. And on the other hand there are systems or methods that can be used in the operating theatre. Systems for lab use are very complex and there is a risk of contamination of the scaffolds. This is the reason why these systems cannot be used in the operating theatre. These kinds of bioreactors are used for tissue engineering applications. The advantage of these systems is the continuous medium flow through the scaffolds. An optimized nutrient supply of the cells and removal of metabolism products are warranted. The scaffold is fixed in a little chamber and the fluid flows continuously through the scaffold. The fluid flow is generated by a pump [9].

A method used in the operating theatre is the manual wetting of the scaffold with fluid containing cells (e.g. blood or bone marrow). The "chronOS Perfusion Concept" (Synthes corp.) is a system which is usable in the operating theatre. The fluid containing body's own cells (autologous bone marrow) is squeezed through the scaffold, fixed in an adapter in front of a syringe, by moving the piston of the syringe. By moving the piston for several times the air in scaffold will be replaced with cell containing fluid.

The developed cell seeding chamber combines the advantages of the systems for lab use and the systems/methods for use in the operating theatre. The cell seeding chamber is a closed assembly. The complete functionality was integrated in a compact chamber. The chamber induced a continuous flow through the scaffold, so the scaffold will be initially seeded with cells. The fluid flow is driven by gravity. There is no need for a pump. The fluid flow should nearly be homogeneous. A volume of $15 \mathrm{ml}$ should flow through the scaffold in a range of 5 to $10 \mathrm{~min}$. By turning the chamber $180^{\circ}$ the process restarts.

\section{$2 \quad$ Materials and Methods}

\section{1 Cell seeding chamber}

The cell seeding chamber is depicted in image 1 . There is a closed chamber (1). Inside of the chamber there are two reservoirs (2). The scaffold is arranged between these two reservoirs. The fluid containing the cells is provided in one of these reservoirs. Now the whole assembly will be positioned in a way that the reservoir containing the fluid is on top of the fixed scaffold (6). The fluid flows out of the reservoir, driven by gravity. The fluid drips on the fixed scaffold and flows through the scaffold. The cells in the fluid adhere to the surface of the scaffold during this process. The fluid flow is adjusted by the dimensions of the reservoir (diameter/height) and the outflow channel (3). The fluid flows through the openings (4) and is collected in the lower reservoir. At the time when the upper reservoir is empty and the whole fluid is collected in the lower reservoir the cell seeding chamber (1) is turned manually by 
$180^{\circ}$ and the process restarts. The fluid is injected via syringe through a septum (7).

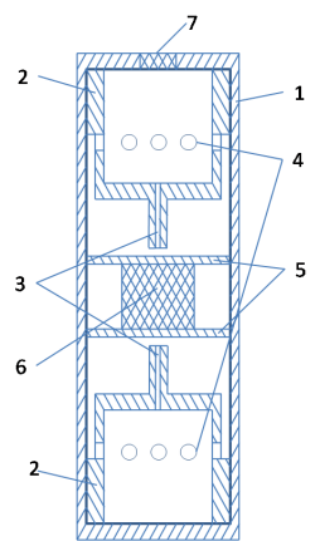

Image 1 Cell seeding chamber

The fluid flow out of the upper reservoir can be divided in three sections. At first there is a flow of a liquid out of a container with a variable fluid level $(h(t))$, see image 2 . The second section is a fluid flow through the channel. And at last there is a drop formation at the end of the channel. The pressure difference which is necessary for the fluid flow is generated by the hydrostatic pressure. The overall height is the result of the fluid level at the container (L1) and the length of the outflow channel (L2). The flow resistance respectively pressure decrease caused by the outflow channel. The length (L2) and inner diameter (d) are the significant parameter. An additional back pressure generated by the drop the end of the channel. The fluid outflow can be described by Torricelli's theorem. The ratio of diameter (D) and height (L1) should as big as possible. So the fluid level variation during time $(\mathrm{L} 1(\mathrm{t}))$ is low and there is a minor deviation in the flow rate.

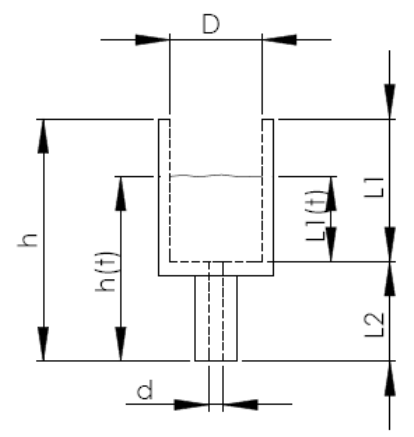

Image 2 Reservoir and channel

\subsection{Blood substitute}

For a better handling and a better reproducibility of the results a blood substitute has been used. The blood substitute should simulate the shear thinning behavior of human blood with a haematocrit of 0.45 at a temperature of $37^{\circ} \mathrm{C}$. Non-newtonian blood substitutes are often based on polymer solutions, for example mixtures of water and glycerin or mixtures of water, glycerin and xathan. Sometimes solu- tions of polyacrylamide are used [3][4][5]. For testing the basic functionality of the cell seeding chamber a solution of Water an Xanthan (0,05 ma\%) has been chosen [5]. The viscosity of the blood substitute was checked with a rheometer Mars 2 (Haake).

The fluid for the medical application of the cell seeding chamber is a non-newtonian fluid (e.g. blood or bone marrow). It is important to adjust the viscosity of the blood substitute in relation to the flow rate in the cell seeding chamber. The mean flow rate has been determined by experiments with blood substitute. There is a low flow rate, so we have a laminar flow. For a rough estimate of the flow rate at the outflow channel a parabolic velocity profile has been assumed. The media flow rate at the outflow channel was calculated out of volumetric flow and cross section area of the outflow channel. Thereby volumetric flow rate and the filling volume of the fluid were given parameters.

\subsection{Outflow channel - hollow needle (stainless steel)}

At first hollow needles made of stainless steel was used as outflow channels. Different hollow needles with a length of $22 \mathrm{~mm}$ have been chosen (17-, 18- and 19-gauge needle).

\subsection{Outflow channel - hose spiral}

Since the inner diameters of the hollow needles are small, there is the risk of an obstruction. Because of this risk a hose spiral used as an alternative outflow channel. The inner diameter of the outflow channel has been significantly enlarged to avoid obstructions and guarantee an undisturbed fluid flow. To generate the necessary flow resistance the outflow channel also has to be significantly lengthened according to Hagen-Poiseuille-law. The outflow channel formed like a spiral because of the increased length. Otherwise the dimension of the whole assembly would become too large. The spiral is based on a hose of PTFE because of the simple machining, advantageously material properties and biocompatibility of this material. Hoses with inner diameter of $\mathrm{D}_{\mathrm{i}}=1.4 / 1,5 / 1,6 \mathrm{~mm}$ have been used for experiments. The length of the hose is approximately $320 \mathrm{~mm}$ the diameter of the winding is $12 \mathrm{~mm}$. There is a vertical inlet and outlet.

\subsection{Experimental analysis}

Experiments have been done to analyze the media flow rate of the reservoir in combination with the hollow needles and the reservoir in combination with a hose spiral (image 3). The reservoir filled with a defined volume of blood substitute $(15 \mathrm{ml})$. The time the fluid needs to flow out of the reservoir has been measured (image 3). To keep the failure small the experiment repeated at least five times. The reservoir and hose spiral cleaned and dried after each test, to ensure the repeatability of the test. 


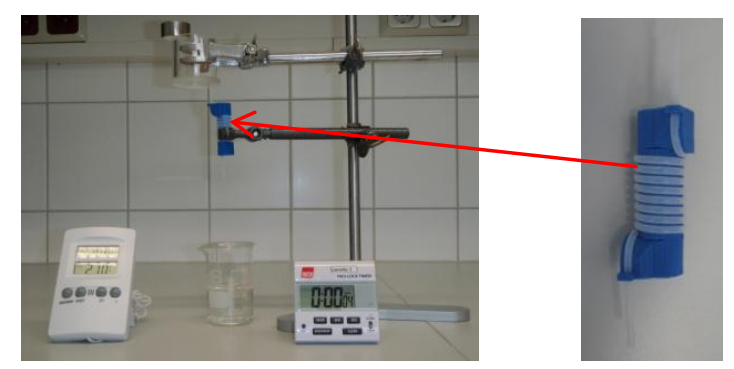

Image 3 Experimental analysis of flow rate - hose spiral

\subsection{Experimental analysis - Scaffold}

A first application for the cell seeding chamber is the vitalization of bone graft substitutes. Experiments with commercially available scaffolds have been done to show the basic functionality of the concept. The xenogenic scaffold Tutobone ${ }^{\circledR}$ (Tutogen) has been used (image 4). It is a material based on bovine cancellous bone. The experimental analysis has been done with samples for laboratory use only.

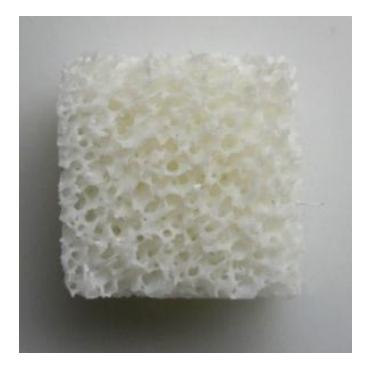

Image 4 Scaffold - cancellous bone (bovine)

To show the perfusion of the scaffolds the blood substitute has been dyed in red with food coloring (A E 124). The cell seeding chamber was manually turned five times to induce the fluid flow through the scaffold for the same number. After that the scaffold was cut into two halves.

\section{Results}

\subsection{Results - hollow needle}

The results of the experiments with blood substitute are insufficient. The media flow rate and the starting behavior of the fluid flow are unsatisfactory. Obstruction of the fluid flow has been detected at all of the tested needles. The reason for these obstructions is probably the drying and deposition of the blood substitute inside the needle.

\section{2 Results - hose spiral}

The experimental results for the different hose spirals and a reservoir with an inner diameter of $30 \mathrm{~mm}$ are shown in tab. 1. The filling volume was $15 \mathrm{ml}$ of blood substitute. The highest media flow rate was determined at the hose spiral with the biggest inner diameter. A nearly continuous media flow rate was detected. An uncomplicated starting behavior was observed.
Table 1: Media flow rate for different tubes

\begin{tabular}{|l|l|l|l|l|}
\hline $\mathrm{D}_{\mathrm{i}}[\mathrm{mm}]$ & $\begin{array}{l}\text { No. of } \\
\text { coils }\end{array}$ & $\begin{array}{l}\text { Diameter } \\
\text { of coil } \\
{[\mathrm{mm}]}\end{array}$ & $\mathrm{t}[\mathrm{min}]$ & $\begin{array}{l}\dot{V}_{\mathrm{m}} \\
{[\mathrm{ml} / \mathrm{min}]}\end{array}$ \\
\hline 1,4 & 8,5 & 12 & 3,48 & 4,31 \\
\hline 1,5 & 8,5 & 12 & 3,41 & 4,39 \\
\hline 1,6 & 8,5 & 12 & 2,29 & 6,55 \\
\hline
\end{tabular}

\subsection{Results - scaffold}

The cut scaffold was analyzed qualitatively by visual inspection. The homogeneous coloring of the scaffolds, in particular in the middle of them, shows that the fluid has been completely dispersed at scaffold (image 5). The basic functionality of the cell seeding chamber has been shown.

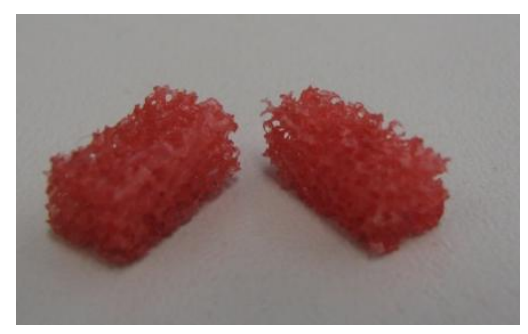

Image 5 Scaffold - dyed

\section{Conclusion}

The experimental analysis has demonstrated that a relative huge inner diameter of the outflow channel is necessary for functionality. To generate the necessary flow resistance the length of the channel had to be enlarged. For easy handling there is a requirement for compact assembly. This is the reason why the outflow channel was formed like a spiral. An advantageously design is given by a hose spiral of PTFE. A preferred design of the cell seeding chamber could be determined (image 6).
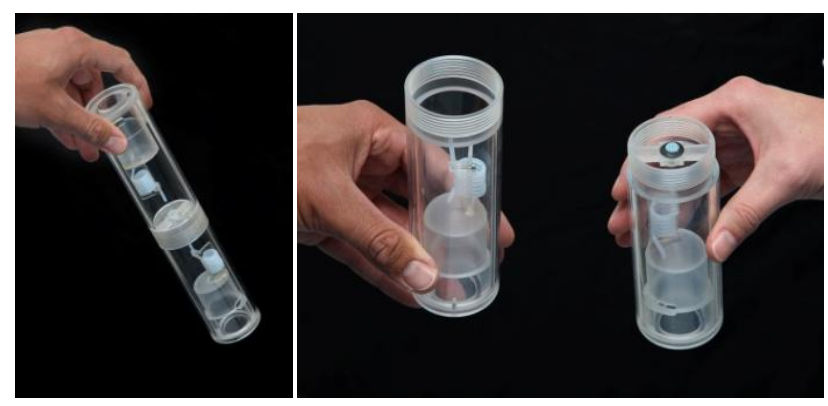

Image 6 Cell seeding chamber - sample for laboratory use

The basic functionality of the cell seeding chamber could be demonstrated with blood substitute. The flow rate of the blood substitute could be adjusted in the range of 4,3 and $6,5 \mathrm{ml} / \mathrm{min}$.

The advantage generated by the cell seeding chamber in relation to established methods have to verify by biological experiments. If it is possible to verify a higher cell seeding 
efficiency, in particular in the middle of the scaffold, there is a basis for an animal experiment. The animal experiment has to demonstrate that the healing behavior of a scaffold vitalized by the cell seeding chamber is better than of a scaffold treated with conventional methods.

\section{References}

[1] Schneider, R.K./Neuss, S./Knüchel, R./Perez-Bouza, A. (2010): Mesenchymale Stammzellen für das "tissue engineering" des Knochens, Der Pathologe, 31: 138146

[2] Poander, S. (2009): In-vitro- und In-vivoUntersuchungen zur Biofunktionalisierung von Knochenersatzmaterialien, Dissertation, FriedrichAlexander-Universität Erlangen-Nürnberg

[3] Gray, J.-D./Owen, I./Escudier, M.P. (2007): Dynamic scaling of unsteady shear-thinning non-Newtonian fluid flows in a large-scale model of a distal anastomosis, Exp Fluids, 43: 535-546

[4] Shibeshi, S.S./Collins, W.E. (2005): The Rheology of Blood Flow in a Branched Arterial System, Appl. Rheol., 15/6: 398-405

[5] Sousa, P.C./Pinho, F.T./Oliveira, M.S.N./Alves, M.A. (2011): Extensional flow of blood analog solutions in microfluidic devices, Biomicrofluidics, 5/014108: 119

[6] Wagner, W. (1997): Strömung und Druckverlust, 4. überarbeitete und erweiterte Auflage, Vogel, 3-80231720-3

[7] Du Plessis, J.P./ Fourie, J.G. (2002): Pressure drop modelling in cellular metallic foams, Chemical Engineering Science, 57: 2781 - 2789

[8] Drosse, I./Volkmer, E./Capanna, R./De Biase, P./ Mutschler, W./Schieker, M. (2008):Tissue engineering for bone defect healing: An update on a multicomponent approach, Injury, Int. J. Care Injured, 39S2, S9-S20

[9] Minuth, W. W./Sittinger, M./Kloth, S. (1998): Tissue engineering: generation of differentiated artificial tissues for biomedical applications, Cell Tissue Res, 291:1-11

[10] Linhart, W./ Briem, D. (2001): Knochenersatz 2000 bis 2010, Der Orthopäde, 30:189-192 